\title{
Metodología de selección de especies de arbolado para el sombreado urbano en la parte oriental de la cornisa Cantábrica
}

\section{Methodology of selection of tree species for urban shading in the eastern part of the Cantabrian coast}

$\underline{\text { I. Azcarate Mutiloa }}^{(*)}$, J.Á. Acero Alejandro ${ }^{(* *)}$, J. Arrizabalaga Ibarzabal $^{(* *)}$

RESUMEN

La mitigación del efecto Isla de Calor es uno de los mayores retos del futuro en las ciudades debido al cambio climático. Uno de los métodos para conseguir el enfriamiento de los espacios urbanos es la atenuación de radiación solar proporcionada por el arbolado. En el presente artículo se plantea una metodología de selección de especies arbóreas para la mejora del confort térmico en zonas urbanizadas de la parte oriental de la cornisa cantábrica. Partiendo de una preselección de especies autóctonas, se obtiene una clasificación de diversas especies aptas para el sombreado describiéndose sus principales características y su posible ámbito de utilización en función de la tipología de espacio urbano.

Palabras clave: Isla de calor, cambio climático, espacio urbano, radiación solar, sombreado, especies de arbolado.

\section{ABSTRACT}

The mitigation of the Urban Heat Island is one of the biggest future challenges in cities due to climate change. One of the methods to achieve the cooling of urban spaces is the attenuation of solar radiation provided by the trees. In the present article, a methodology of selection of arboreal species is proposed for the improvement of thermal comfort in urbanized areas of the eastern part of the Cantabrian coast. Starting from a pre-selection of native species, a classification of various species suitable for shading is obtained, describing its main characteristics and its possible use scope according to the typology of urban space.

Keywords: Urban heat island, climate change, urban space, solar radiation, shading, tree species.

(*) Departamento de Arquitectura. Universidad del País Vasco/Euskal Herriko Unibertsitatea (España).

(**) Energy and Environmental Division, Tecnalia. Bizkaia (España).

Persona de contacto/Corresponding author: inigo.azcarate@ehu.eus (I. Azcarate Mutiloa)

ORCID: https://orcid.org/o0oo-0002-0207-624X (I. Azcarate Mutiloa); https://orcid.org/oooo-0001-6214-0605

(J.A. Acero Alejandro); https://orcid.org/oooo-0003-1128-7230 (J. Arrizabalaga Ibarzabal)

Cómo citar este artículo/Citation: Azcarate Mutiloa, I.; Acero Alejandro, J.A.; Arrizabalaga Ibarzabal, J. (2019). Metodología de selección de especies de arbolado para el sombreado urbano en la parte oriental de la cornisa Cantábrica. Informes de la Construcción, 71(556): e322. https://doi.org/10.3989/ic.65135

Copyright: (C) 2019 CSIC. Este es un artículo de acceso abierto distribuido bajo los términos de la licencia de uso y distribución Creative Commons Reconocimiento 4.o Internacional (CC BY 4.0). 


\section{INTRODUCCIÓN: EL ARBOLADO EN EL ENTORNO URBANO}

\subsection{La isla de calor y el cambio climático}

El efecto conocido como isla de calor (UHI- Urban Heat Island) es una situación que se siente con mayor intensidad dentro de las ciudades, consistente en la dificultad para disipar el calor generado y acumulado en áreas urbanas. Generalmente, este efecto es más notable en horas nocturnas cuando en el entorno rural el enfriamiento es más rápido (1).

Los materiales utilizados tradicionalmente para la construcción urbana presentan unos valores de albedo (porcentaje de radiación solar que cualquier superficie refleja respecto a la radiación que incide sobre la misma) menores que los existentes en el medio rural, por lo que la absorción de energía por parte de éstos materiales ocasiona un mayor almacenamiento de calor en los mismos. Como consecuencia, y en función de sus valores de emisividad térmica (capacidad de un material para liberar el calor absorbido a través de radiación infrarroja), la difusión de calor por parte de dichos materiales será de mayor magnitud por lo que el enfriamiento del ambiente resultará más dificultoso.

Se prevé que en un futuro las ciudades van a ir experimentando un aumento de la temperatura debido a los efectos del cambio climático, con la consecuente repercusión en el confort térmico, por lo que la mitigación del efecto isla de calor es uno de los mayores retos del futuro (2). Algunos de los métodos para conseguir el enfriamiento del entorno urbano son la utilización de materiales adecuados, un nivel de ventilación que permita eliminar el calor acumulado, el incremento de zonas verdes urbanas, y el sombreado (1).

\subsection{Beneficios de las zonas verdes y el arbolado}

Los beneficios de carácter ambiental que ofrecen las zonas verdes y el arbolado son la mejora del confort térmico $(3,4,5)$ y la posible reducción de contaminación ambiental, mejorando las características del aire urbano $(6,7)$, aunque también pueden generar una atenuación de la velocidad del viento (8) reduciendo los niveles de ventilación de las calles, lo cual tiene una repercusión negativa en el confort térmico.

Otros beneficios de carácter social son la mejora de la salud física del individuo posibilitando el desarrollo de actividades físicas o lúdicas $(7,9,10)$, y el bienestar mental derivado de las cualidades estéticas naturales y la generación de lugares de referencia para la sociedad (7).

\subsubsection{El confort térmico. La radiación solar y la temperatura media radiante}

La radiación presente en el medio urbano se puede distinguir en:

- Radiación de onda media y corta: se corresponde con la radiación solar.

- Radiación de onda larga: radiación reemitida por los materiales encontrados en la atmósfera o por la propia tierra.

El confort térmico depende de diversos factores y se puede medir mediante diferentes índices, siendo uno de los más utilizados el índice PET (Physiologically Equivalent Tempe- rature) derivado del modelo de balance térmico del cuerpo humano MEMI (11). También existen otros métodos de medida como pueden ser el índice PMV (Predicted mean Vote) (12) el cual refleja la opinión de un grupo numeroso de personas sobre su sensación térmica estableciendo siete niveles de confort (de $-3 \mathrm{a}+3$ ), o el PPD (Percentage of People Dissatisfied) el cual guarda correspondencia con el índice anterior. A su vez, el índice CP (Cooling Power) establece diez niveles de confort en función de la temperatura ambiente media y la velocidad del viento (13).

Para el cálculo del índice PET se toma en consideración información de carácter meteorológico, aunque también se requiere información de las características fisiológicas de las personas (edad, sexo, altura, peso, etc.). Algunos de los parámetros meteorológicos considerados para el cálculo de índice PET son la temperatura del aire, la humedad relativa, la velocidad de viento o la temperatura media radiante. Ésta última determina la medida del calor generado por medio de los materiales en el entorno urbano como consecuencia de la radiación solar, resultando ser el parámetro más relevante para el confort térmico y el índice PET especialmente en días despejados (14).

El concepto de temperatura media radiante se define como "la temperatura uniforme en un recinto imaginario en el que la transferencia del calor radiante desde el cuerpo humano iguala la transmisión del calor radiante en dicho recinto no uniforme". (15). Está demostrado que la diferencia entre la temperatura media radiante de una zona expuesta al sol y la de otra en sombra podría alcanzar los $25^{\circ} \mathrm{C}$ (11).

\subsubsection{El arbolado y el sombreado}

Cualquier elemento urbano apto para generar sombreado es capaz de reflejar, difundir y absorber radiación solar. Las hojas de los árboles en general son capaces de reflejar el 10\% de la energía visible y el 50\% de la energía solar infrarroja, mientras que pueden absorber el $80 \%$ de la energía visible y el $20 \%$ de la energía solar infrarroja. En consecuencia, la radiación transmitida resultante a través de sus hojas consistiría en un 10\% de la energía visible más un 30\% de la energía solar infrarroja (16). La magnitud de la atenuación solar varía en función de cada especie de arbolado y sus características estructurales, como son la densidad de foliación, la altura, el diámetro de la copa o la proximidad entre elementos $(17,18)$.

La efectividad del sombreado generado por los árboles para la mejora del confort térmico disminuye con el aumento de la profundidad de los espacios urbanos, es decir, cuando la proporción entre la altura de los edificios y la anchura de las calles es mayor (18).

\subsubsection{Las especies autóctonas}

Se consideran especies autóctonas aquellas que han existido en un lugar o ecosistema concreto previamente a cualquier intervención humana. Ello se debe a que las condiciones para el desarrollo de dichas especies se satisfacen en un lugar geográfico concreto, en función de los aspectos climatológicos, geológicos o de entorno natural. (19)

Aunque algunas especies no sean consideradas autóctonas, cabe la posibilidad de que pudieran catalogarse como asimiladas; aunque su implantación en un lugar concreto se haya 
debido a la acción del hombre, sus requerimientos de desarrollo son adaptables al nuevo medio sin afectar al resto de flora y fauna existente.

\subsection{Características de las especies arbóreas}

\subsubsection{Características morfológicas}

Las características geométricas parciales del elemento arbóreo (Figura 1) que influyen en su capacidad de sombreado $(18,20)$ son las siguientes:

(H) Altura total del árbol.

(Ht) Altura del tronco.

(Dc) Diámetro de la copa.

(Hc) Altura de la copa.

La forma de las copas puede asemejarse a una geometría cilíndrica-ovoidal, esférica, o cónica. Para la determinación de la forma, independientemente de su aspecto visual, es posible realizar una clasificación de las especies arbóreas en función de la relación entre el diámetro de la copa en su punto máximo y la altura de la misma (21).

La forma y dimensiones de la copa de los árboles influyen directamente en el área sombreada generada por el mismo. A igualdad de volumen, la cantidad de sombra generada por un árbol de copa ancha (esférica) es considerablemente mayor que la generada por uno de copa estrecha (cilíndrica-ovoidal) cuando el ángulo solar respecto la horizontal se encuentra entre los $55^{\circ}$ y $75^{\circ}$. En cambio, la cantidad de sombra generada por un árbol de copa alta sería algo superior cuando el ángulo solar se encuentra entre $35^{\circ}$ y $45^{\circ}$ (19). Sobre todo en el primer caso, una altura de tronco que posibilite el tránsito peatonal bajo la copa aumenta asimismo el área de pavimento sombreada.

\subsubsection{Características fisiológicas}

En las zonas climáticas donde el sombreado del arbolado resulta solamente necesario en los meses más calientes del año son preferibles las especies de hoja caduca, es decir, aquellas cuyas hojas caen en las estaciones más térmicamente desfavorables del año (otoño- invierno), proporcionando un adecuado sombreado cuando éste es necesario (primavera- verano) aunque sin interferir en la captación solar durante las estaciones frías (22).

Las especies de hoja perenne, dado que sus hojas se mantienen en el árbol durante todas las estaciones, atenúan la radiación solar de manera uniforme durante todo el año, generando un indeseado sombreado cuando las temperaturas son bajas.

\subsubsection{Densidad de foliación y transmitancia solar. Los parámetros LAD y LAI}

La densidad de foliación es un parámetro determinante en la capacidad de sombreado que puede ofrecer una especie de arbolado. El parámetro más utilizado que determina esta característica es el denominado índice de área foliar LAI (leaf area index), el cual es una variable adimensional $\left(\mathrm{m}^{2} /\right.$ $\mathrm{m}^{2}$ ) que fue primeramente definida como el total de superficie (a una cara) del tejido fotosintético de una planta por cada unidad de superficie de suelo en proyección (23). Dicho de otra manera, sería la cantidad de $\mathrm{m}^{2}$ de hoja que debe atravesar la radiación solar por cada $\mathrm{m}^{2}$ que incidirá en el suelo.

El LAI tiene relación directa con otros parámetros como el LAD (leaf area density, $\mathrm{m}^{2} / \mathrm{m}^{3}$ ) que es la superficie de hoja por cada unidad de volumen de la copa arbórea. El LAI depende de la composición de cada especie, la estación del año, o las condiciones microclimáticas de cada lugar, siendo asimismo un parámetro dinámico para cada especie, que varía día a día, principalmente en primavera y otoño en las especies de hoja caduca, o incluso año a año (24).

Existen otros parámetros que pueden determinar la cantidad de radiación solar que atraviesa el foliación o la cobertura vegetal de un árbol, como son el factor de sombra (nivel de atenuación solar) o el rango de transmitancia, los cuales expresan la misma magnitud aunque de manera inversa.

Lógicamente existe una relación proporcional entre el LAI y el factor de sombra de un árbol; cuanto más elevado es el índice LAI (el cual varía entre 3 y 6 para árboles considerados aptos para el sombreado) mayor será el factor de sombra del mismo, haciendo posible la incidencia de una menor
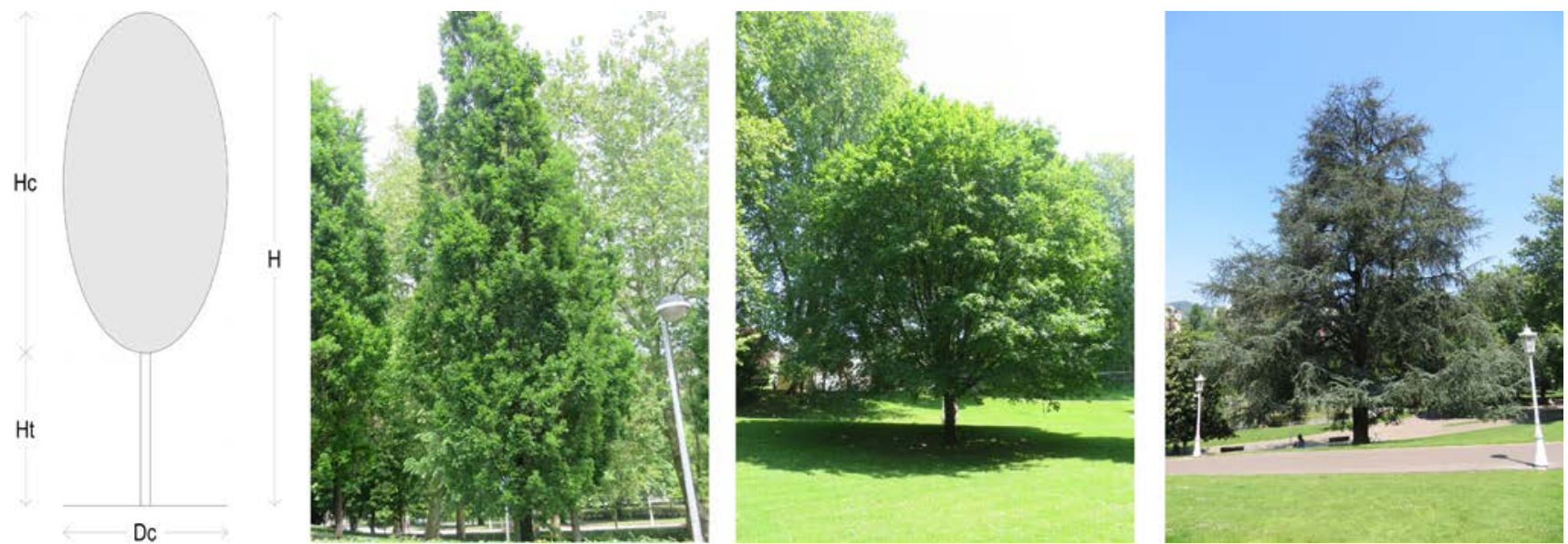

Figura 1. Izquierda. Esquema parámetros geométricos del árbol. Derecha. Forma de las copas Quercus robur (Cilíndrica-Ovoidal). Acer platanoides (Esférica). Cedrus atlantica (Cónica). 
cantidad de radiación solar por cada unidad de superficie de pavimento. El parámetro LAI se convierte en un indicador fundamental para evaluar la capacidad de mejora de las condiciones de confort térmico del arbolado urbano (18).

\subsubsection{Otras características no relacionadas con el sombreado}

Independientemente de las cualidades que presenten los árboles para las funciones de sombreado, éstos deben poder adaptarse a las diversas condiciones del entorno urbano en el cual van a habitar. En este sentido, se consideran los siguientes condicionantes específicos para la elección de especies en función de su adaptación al entorno:

- Tolerancia al calor: resistencia a las altas temperaturas.

- Tolerancia a la 'pavimentación', donde las tierras presentan una mayor compacidad dificultando el enraizamiento.

- Tolerancia a la salinidad: en caso de ubicaciones cercanas al litoral.

Asimismo, la plantación de arbolado en los entornos urbanos puede generar también efectos negativos sobre la población (25). Las principales desventajas son las siguientes:

- Daños físicos o materiales causados por caídas de hojas, frutos o ramas sobre las personas, propiedades o infraestructuras.

- Repercusión económica de las labores de mantenimiento de los elementos arbóreos así como de sus partes desprendidas.

- Excesiva atenuación lumínica en los espacios públicos abiertos y las edificaciones, además de reducción de ventilación de los espacios urbanos delimitados.

\subsection{Características del entorno urbano}

Las posibilidades de mejora del confort térmico dependen de la anchura del espacio no edificado (calle) y su proporción con la altura de los edificios que las delimitan, además de las características del acabado superficial $(26,27,28)$. Por consiguiente, la elección de las especies de arbolado se verá asimis- mo condicionada por las características del espacio urbano en el cual se vayan a ubicar.

Se realiza una clasificación de calles en función de su anchura:

- Calles estrechas: anchura $\leq 12 \mathrm{~m}$.

- Calles medianas: $12 \mathrm{~m} \leq$ anchura $\leq 20 \mathrm{~m}$.

- Calles anchas: $20 \mathrm{~m}$. $\leq$ anchura $\leq 30 \mathrm{~m}$.

- Espacios abiertos: espacios urbanos con anchura superior a los $30 \mathrm{~m}$.

Se deben considerar también los siguientes parámetros:

AR (Aspect Ratio): relación entre la altura de las edificaciones que delimitan la calle y anchura de la misma ( $\mathrm{He} / \mathrm{A}$ ) (Figura 2). Cuanto mayor es el ratio AR, la superficie de calle afectada por el sombreado de las edificaciones será también mayor (29), aunque el atrapamiento de la radiación de onda larga reemitida por los diferentes materiales será también de mayor magnitud.

SVF (Sky View Factor): se define como la fracción de cielo visible desde un punto concreto de la calle. Un elevado valor SVF conlleva un bajo nivel de sombreado, lo que causará un deficiente confort térmico en verano, mientras que un elevado valor SVF producirá el mismo efecto no deseado durante el invierno $(26,27,28)$.

Orientación del eje de la calle: condicionará directamente la superficie afectada por el sombreado de los edificios durante las distintas horas del día. Su tipificación se efectúa en función del ángulo en planta que formaría el eje de la calle con la línea Norte-Sur geográfica $(26,27,28)$.

Considerando que las horas centrales del día coinciden con los momentos de mayor radiación solar y que los espacios peatonales tienden a situarse cercanos a las fachadas de los edificios, una orientación de la calle Norte-Sur posibilitará una mayor incidencia de la radiación solar en la totalidad de la superficie de la calle en dichas horas centrales, mientras

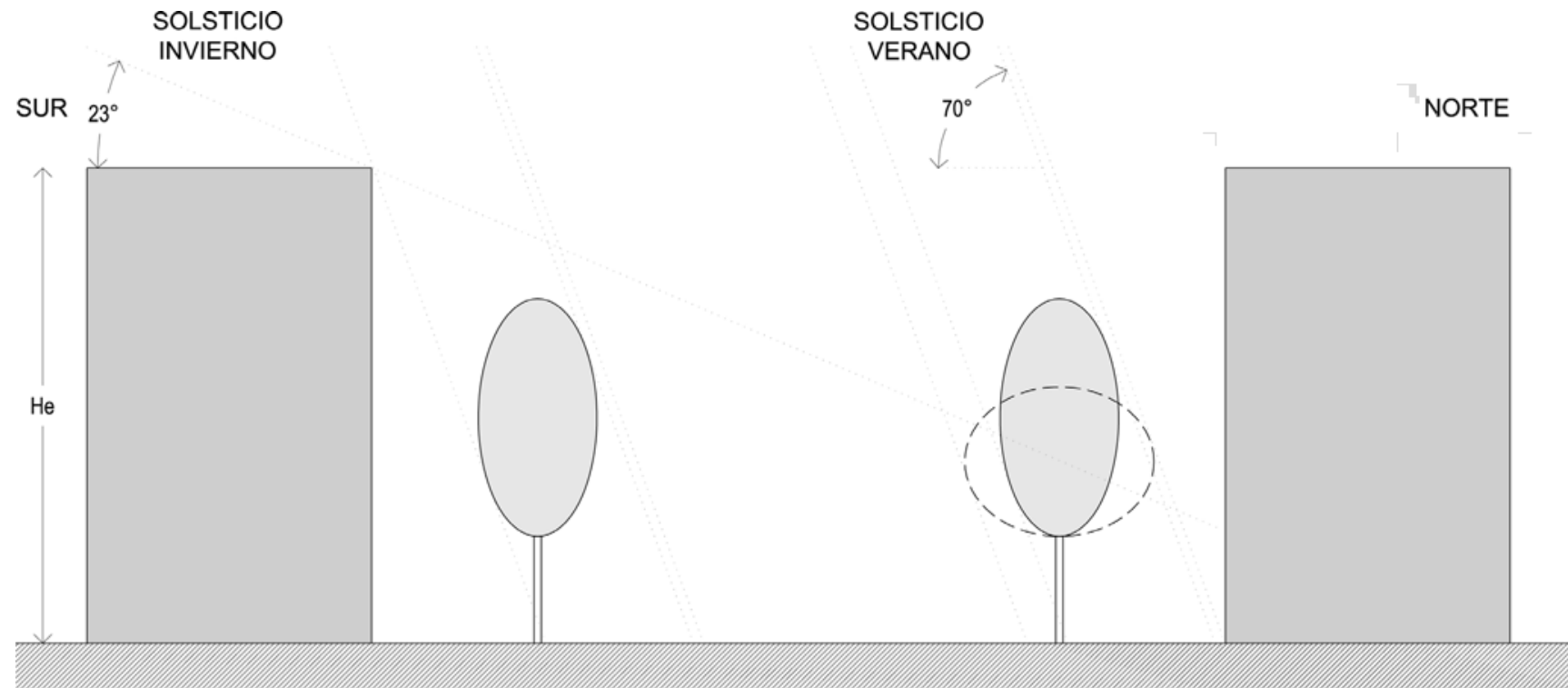


que en una orientación de la calle Este-Oeste la incidencia será mayor en la parte Norte de la misma.

Teniendo en cuenta que una forma de copa ancha proporción una mayor superficie sombreada cuando el ángulo solar respecto la horizontal es alto (19), una calle de orientación Norte-Sur requerirá especies de copa ancha a ambos lados de la calle, mientras que una calle de orientación EsteOeste podrá disponer de arbolado de copa más estrecha, sobre todo en el lado Sur de la misma, siempre que resultara necesario.

\subsection{Objetivos del presente estudio}

En el presente estudio se pretende plantear una metodología de selección de especies arbóreas para su plantación en zonas urbanizadas de cara a mejorar el confort térmico de la población, determinando los principales criterios de selección y condicionantes que influyen en su capacidad de sombreado (30).

Posteriormente, se procede a realizar una selección de especies arbóreas para su utilización en los espacios urbanos de la parte oriental de la cornisa cantábrica, determinándose sus principales parámetros de carácter morfológico y fisiológico, añadiéndose asimismo sus características de adaptación al entorno urbano. Se plantea además su posible ámbito de utilización según las distintas tipologías de espacios urbanos contemplados.

\section{CONDICIONES MICROCLIMÁTICAS: EL CLIMA EN LA PARTE ORIENTAL DE LA CORNISA CANTÁBRICA}

Los principales factores que determinan la climatología de las regiones de la parte oriental de la cornisa cantábrica son su latitud (regiones situadas en latitudes medias), su reducida distancia al mar (costas están bañadas por el mar Cantábrico) y su complejo relieve orográfico. A su vez, estas regiones van variando sus características meteorológicas pudiéndose distinguir tres subregiones climáticas en función de su distancia a la línea de costa:

- Vertiente Atlántica: temperaturas moderadas en invierno $\left(\mathrm{o}-10^{\circ} \mathrm{C}\right)$ y verano $\left(18-25^{\circ} \mathrm{C}\right)$, muy lluviosas $(1.200$ - $2.000 \mathrm{~mm} /$ año) y con gran influencia oceánica.

- Zona intermedia de transición: clima intermedio entre el clima oceánico y mediterráneo.

- Zona de interior: veranos secos y calurosos $\left(25-35^{\circ} \mathrm{C}\right)$ e inviernos fríos $\left(-5-5^{\circ} \mathrm{C}\right)$. Pluviosidad relativamente débil (aproximadamente 500 - $800 \mathrm{~mm} /$ año).

Se prevé que el calentamiento global multiplique los fenómenos climáticos extremos (inundaciones, sequías, olas de calor y de frío) agravando los procesos de desertificación y erosión.
La península ibérica, debido a su situación geográfica, es uno de las zonas europeas más vulnerables a priori, pudiendo afectar muchos de estos impactos a su vertiente Norte.

\section{METODOLOGÍA PARA LA SELECCIÓN DE ESPECIES}

\subsection{Metodología}

La metodología para la selección (o descarte) de especies de arbolado para el sombreado urbano consiste en un proceso donde las características de cada especie de arbolado se van sometiendo a distintos requisitos para valorar la aptitud de cada una para la función de sombreado prevista.

La secuencia metodológica propuesta comprende los siguientes pasos (Figura 3).

Paso 1. Preselección de especies autóctonas y asimiladas: Se realiza una primera preselección de aproximadamente 60 especies de arbolado $(31,32)$.

Paso 2. Características fisiológicas - Condicionantes climatológicos: Aunque en general se consideran más adecuadas las especies de hoja caduca (22), no se descarta la posibilidad de utilizar especies de hoja perenne en espacios abiertos.

Si bien no tiene relación directa con su capacidad de sombreado, se toma en consideración si la especie es invasora, es decir, si se encuentra incluida en el Real Decreto 630/2013 por el que se regula el catálogo español de especies exóticas invasoras.

Paso 3. Densidad de foliación - Radiación solar: Ya que en el presente estudio no se procede a cuantificar pormenorizadamente los valores de LAD, LAI o transmitancia de todas las especies, se consideran adecuadas para el sombreado aquellas especies que presenten una densidad de hojas media o alta.

En la Tabla 1 se muestra el baremo relacional contemplado a modo de referencia entre la densidad de foliación y los parámetros LAI de las especies, incluyéndose algunas de ellas a modo de referencia $(20,33)$.

Paso 4. Características morfológicas: Se contemplan las características geométricas o formales que repercutan en la capacidad de sombreado de las especies.

Tabla 1. Baremo densidad de foliación - parámetro LAI.

\begin{tabular}{|l|l|l|}
\hline $\begin{array}{c}\text { Densidad } \\
\text { de foliación }\end{array}$ & LAI $\left(\mathbf{m}^{2} / \mathbf{m}^{2}\right)$ & \multicolumn{1}{|c|}{ Ejemplos especies } \\
\hline Baja & $\mathrm{LAI}<3, \mathrm{O}$ & Larix decidua - Pinus pynaster \\
\hline Media & $3, \mathrm{O}<\mathrm{LAI}<5, \mathrm{O}$ & Betula alba - Quercus ilex \\
\hline Alta & $\mathrm{LAI} \geq 5,0$ & $\begin{array}{l}\text { Fagus sylvatica }- \text { Platanus } \\
\text { hispánica }\end{array}$ \\
\hline
\end{tabular}

Tabla 2. Criterios para la elección del ámbito de utilización.

\begin{tabular}{|l|c|c|c|c|}
\hline & H,max. & Cr. med. & Foliación & Adaptación pavimento \\
\hline Calles estrechas & $\leq 10 \mathrm{~m}$. & $\leq 6 \mathrm{~m}$. & Caduca $\left.*^{*}\right)$ & Media o alta \\
\hline Calles medianas & $\leq 25 \mathrm{~m}$. & $\leq 15 \mathrm{~m}$. & Caduca & Indiferente \\
\hline Calles anchas & $\leq 30 \mathrm{~m}$. & $\leq 20 \mathrm{~m}$. & Caduca & Indiferente \\
\hline Espacios abiertos & Indiferente & Indiferente & Caduca o perenne & Indiferente \\
\hline
\end{tabular}

$(*)$ Ante la escasez de especies para aceras estrechas se admiten especies de hoja perenne. 


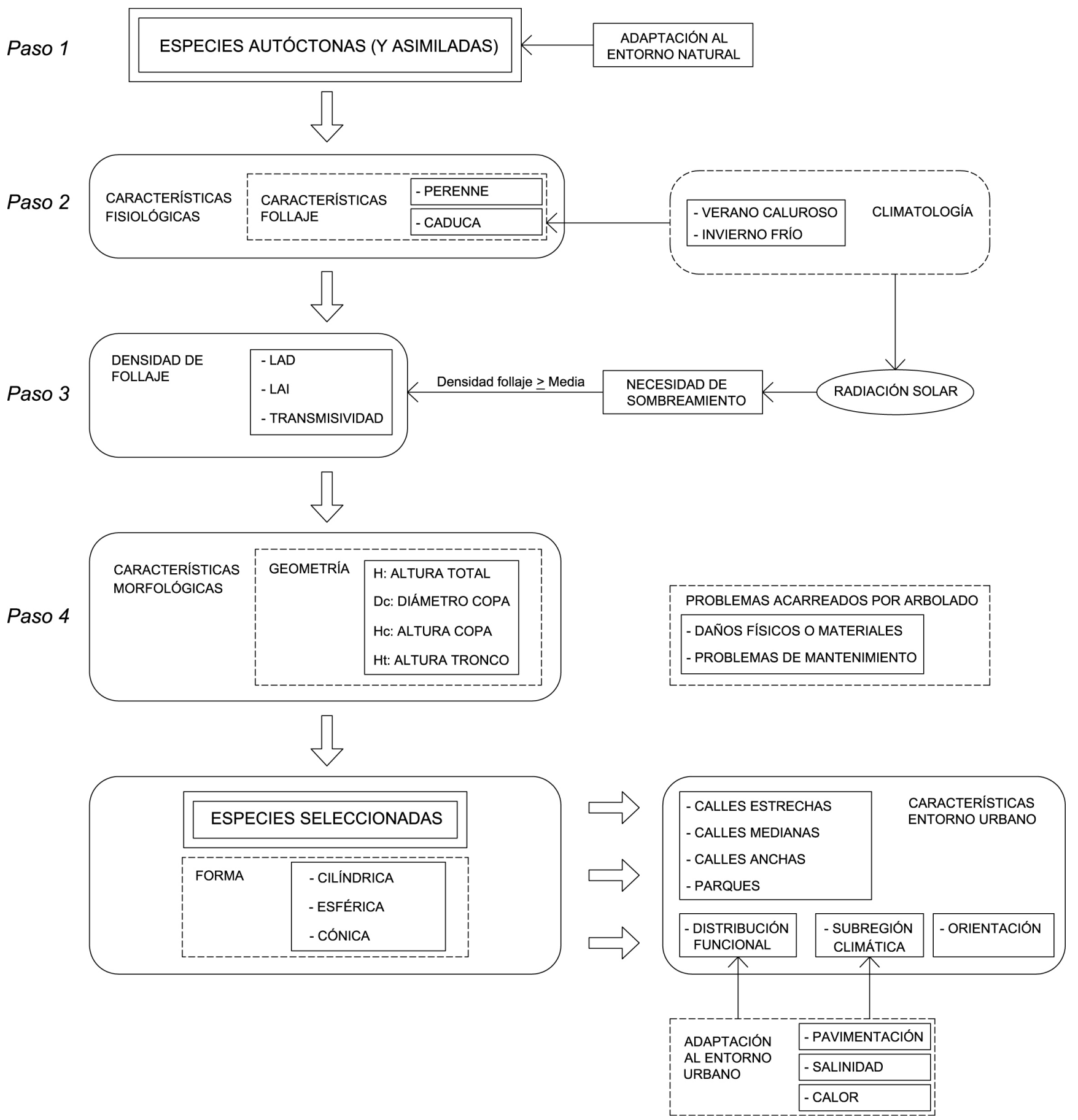

Figura 3. Secuencia metodológica para la selección de especies de arbolado urbano.

Una excesiva altura de los árboles, en función de la forma de su copa así como de la anchura y orientación de la calle, generará sombra no deseada en las fachadas de los edificios afectando negativamente en la iluminación natural de los espacios interiores (21). Se considera una morfología inadecuada por altura excesiva cuando la relación entre la anchura media de la copa y la altura media del árbol es inferior a o,3 (Dc.med / H.med < 0,3).

Se toma asimismo en consideración una altura insuficiente en función de la altura de tronco debido a su repercusión en el área sombreada resultante (19). Por otro_lado, debido a razones de tránsito peatonal, se recomienda una altura libre mínima de tronco de 2,20 metros.
Otras consideraciones no relacionadas con la capacidad de sombreado:

Si bien los siguientes parámetros no se contemplan como criterio de descarte, se toman en consideración como características adicionales de las especies.

- Adaptación al calor, el pavimento y la salinidad: se consideran estas características de comportamiento de las especies en el entorno urbano.

- Problemas acarreados por arbolado urbano: se consideran aspectos como los daños físicos o materiales, así como problemas de mantenimiento que pueda generar el arbolado. 
Los criterios utilizados para la elección del posible ámbito de utilización de cada especie se describen en la Tabla 2, en función de la correspondencia entre sus características geométricas y la anchura de las calles.

\section{RESULTADOS}

\subsection{Especies descartadas}

Los motivos de descarte provienen principalmente de las características morfológicas de las especies, aunque también en menor grado de sus características fisiológicas o su densidad de foliación.

En lo que respecta a las características morfológicas, el descarte de especies se ha realizado principalmente por la altura del árbol: una reducida altura condiciona su capacidad de sombreado mientras que una altura excesiva en proporción a la anchura de su copa, además de generar sombra no desea- da en las fachadas de los edificios, conlleva dificultades para su plantación en calles estrechas o medianas. Sin embargo, una excesiva altura, siempre que la anchura de su copa sea proporcionada según el baremo establecido, no es obstáculo para la disposición de especies en espacios abiertos, excepto cuando exista una razón adicional para su descarte.

Las características fisiológicas que han conllevado el descarte de especies han sido sobre todo la no caducidad de las hojas y la poca densidad de foliación, ya que este aspecto influye directamente en el índice LAI o el coeficiente de transmitancia. Asimismo, varias especies han sido descartadas por considerarse invasoras, es decir, por dificultar el crecimiento de otras especies o generar interferencias en los ecosistemas naturales o urbanos.

En la Tabla 3 se describen las especies y los criterios por los que se han descartado añadiéndose las respectivas observaciones e indicando si adicionalmente presentan otros in-

Tabla 3. Especies de arbolado descartadas. Criterios de descarte.

\begin{tabular}{|c|c|c|c|c|c|c|c|}
\hline \multirow[b]{2}{*}{$\begin{array}{l}\text { Nombre } \\
\text { botánico }\end{array}$} & \multirow[b]{2}{*}{ Nombre común } & \multicolumn{3}{|c|}{ CRITERIOS DE DESCARTE } & \multicolumn{3}{|c|}{ OTRAS CONSIDERACIONES } \\
\hline & & $\begin{array}{l}\text { Características } \\
\text { Morfológicas }\end{array}$ & \begin{tabular}{|c|} 
Características \\
Fisiológicas \\
\end{tabular} & $\begin{array}{c}\text { Densidad } \\
\text { de Foliación }\end{array}$ & Observaciones. & Invasora & $\begin{array}{l}\text { Problemas } \\
\text { acarreados } \\
\end{array}$ \\
\hline Abies alba & Abeto & & $\mathrm{X}$ & & Perenne. & & \\
\hline Acacia dealbata & Mimosa & $\mathrm{X}$ & $\mathrm{X}$ & & $\begin{array}{l}\text { Altura insuficiente. Especie invasora. No } \\
\text { autóctona. }\end{array}$ & $\mathrm{X}$ & $\mathrm{X}$ \\
\hline Ailanthus altissima & Ailanto & & $\mathrm{X}$ & & Especie invasora. No autóctona. & $\mathrm{X}$ & $\mathrm{X}$ \\
\hline Arbutus unedo & Madroño & $\mathrm{X}$ & $\mathrm{X}$ & & Perenne y especie invasora. & $\mathrm{X}$ & $\mathrm{X}$ \\
\hline Ceratonia siliqua & Algarrobo & $\mathrm{X}$ & & & Altura insuficiente. Utilizada para cultivos. & & $\mathrm{X}$ \\
\hline Cormus sanguinea & Cornejo & $\mathrm{X}$ & $\mathrm{X}$ & & Altura insuficiente e invasora. & $\mathrm{X}$ & $\mathrm{X}$ \\
\hline Corylus avellana & Avellano & $\mathrm{X}$ & & & Ramosa desde la base y caída de frutos. & & $\mathrm{X}$ \\
\hline $\begin{array}{l}\text { Crataegus } \\
\text { Monogyna }\end{array}$ & Majuelo & $\mathrm{X}$ & & & $\begin{array}{l}\text { Demasiado ramosa desde la base y } \\
\text { espinosa. }\end{array}$ & & \\
\hline $\begin{array}{l}\text { Cupresus } \\
\text { sempervirens }\end{array}$ & Ciprés & $\mathrm{X}$ & $\mathrm{X}$ & & $\begin{array}{l}\text { Morfología inadecuada. Copa estrecha. } \\
\text { Perenne. }\end{array}$ & & \\
\hline $\begin{array}{l}\text { Elaeagnus } \\
\text { angustifolia }\end{array}$ & Árbol paraíso & $\mathrm{X}$ & & & Demasiado ramosa desde la base. & & $\mathrm{X}$ \\
\hline Eucalytus globulus & Eucalipto blanco & $\mathrm{X}$ & & & Morfología inadecuada. Copa estrecha. & & \\
\hline Ficus carica & Higuera & $\mathrm{X}$ & & & Demasiado ramosa desde la base & & \\
\hline Ilex aquifolium & Acebo & $\mathrm{X}$ & $\mathrm{X}$ & & Especie salvaje protegida. & & \\
\hline $\begin{array}{l}\text { Juniperus } \\
\text { communis }\end{array}$ & Enebro & $\mathrm{X}$ & & & Ramosa desde la base. Caída de frutos. & & $\mathrm{X}$ \\
\hline $\begin{array}{l}\text { Juniperus } \\
\text { oxycedrus }\end{array}$ & Enebro rojo & $\mathrm{X}$ & & & Ramosa desde la base. Caída de frutos. & & $\mathrm{X}$ \\
\hline Larix decidua & Alerce & & $\mathrm{X}$ & $\mathrm{X}$ & $\begin{array}{l}\text { Perenne y baja densidad de foliación. No } \\
\text { autóctona. }\end{array}$ & & \\
\hline Laurus nobilis & Laurel & $\mathrm{X}$ & $\mathrm{X}$ & & Ramosa desde base y perenne. & & \\
\hline Malus sylvestris & Manzano silvestre & $\mathrm{X}$ & & & Ramosa desde base. Utilizada para cultivos. & & $\mathrm{X}$ \\
\hline Olea europaea & Olivo & $\mathrm{X}$ & & & Ramosa desde base. Utilizada para cultivos. & & \\
\hline Pinus pynaster & Pino marítimo & & $\mathrm{X}$ & & $\begin{array}{l}\text { Perenne y poco utilizada en entornos } \\
\text { urbanos. }\end{array}$ & & \\
\hline Populus nigra & Chopo & $\mathrm{X}$ & & & Morfología inadecuada. Copa estrecha. & & \\
\hline Prunus avium & Cerezo & $\mathrm{X}$ & & & Generalmente para cultivos. & & $\mathrm{X}$ \\
\hline Prunus spinosa & Endrino & $\mathrm{X}$ & & & Altura insuficiente y espinosa. & & $\mathrm{X}$ \\
\hline $\begin{array}{l}\text { Robinia } \\
\text { pseudoacacia }\end{array}$ & Robinia & & $\mathrm{X}$ & & Uso extendido aunque invasora. & $\mathrm{X}$ & $\mathrm{X}$ \\
\hline Salix alba & Sauce blanco & $\mathrm{X}$ & & & Ramosa desde la base y no autóctona. & & \\
\hline Salix atrocinerea & Sauce cenizo & $\mathrm{X}$ & & $\mathrm{X}$ & Altura insuficiente y copa poco densa. & & \\
\hline Tamarix gallica & Tamariz & $\mathrm{X}$ & & $\mathrm{X}$ & $\begin{array}{l}\text { Altura insuficiente, poca sombra y no } \\
\text { autóctona. }\end{array}$ & & \\
\hline Taxus baccata & Tejo común & & $\mathrm{X}$ & & Lento crecimiento y partes tóxicas. & $\mathrm{X}$ & $\mathrm{X}$ \\
\hline
\end{tabular}



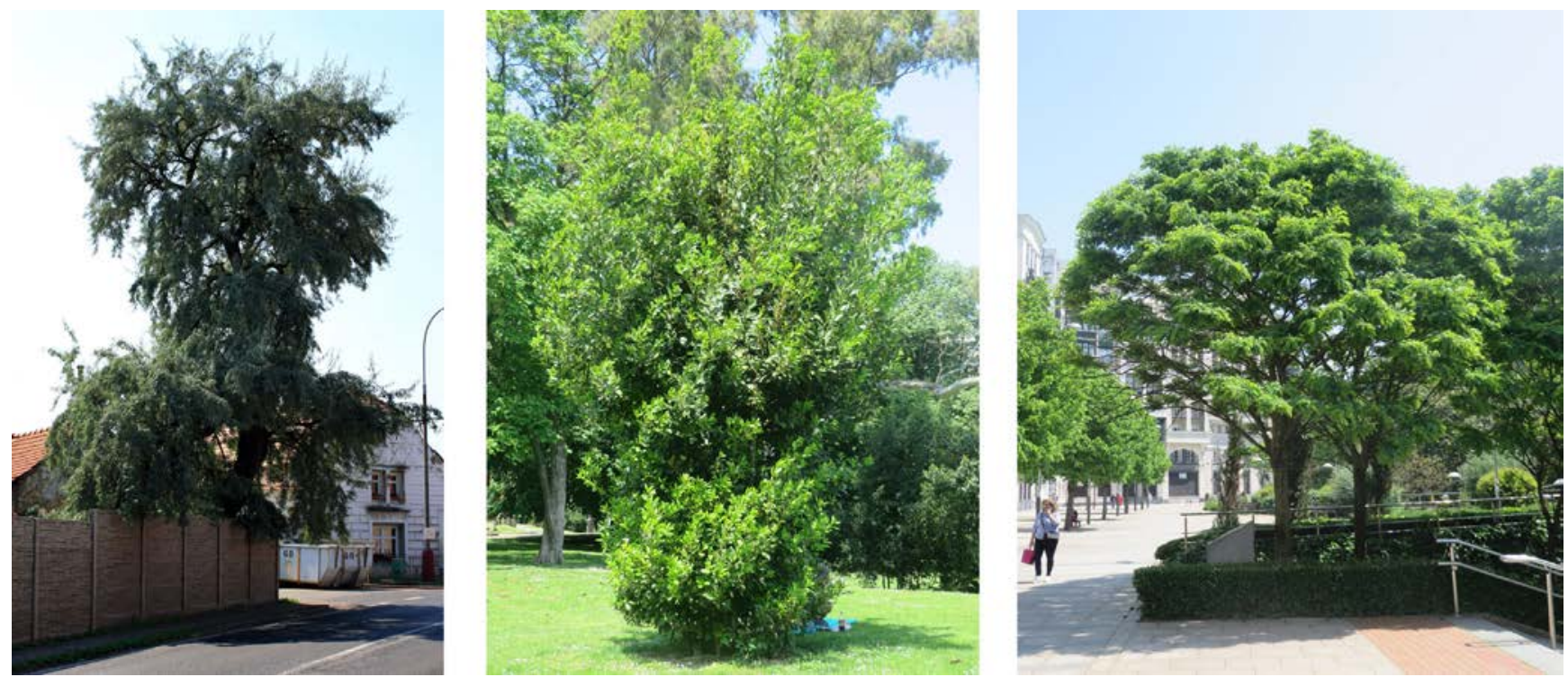

Figura 4. Especies descartadas. Elaeagnus angustifolia, Laurus nobilis. Robinia pseudoacacia.

convenientes para su plantación en entornos urbanos. En la Figura 4 se muestra un ejemplo de algunas de las especies descartadas.

\subsection{Especies seleccionadas}

Las especies que no han sido descartadas se consideran susceptibles de ser utilizadas en los entornos urbanos para funciones de sombreado y consiguiente mejora del confort térmico.

En la Tabla 4 se presenta un listado de las especies seleccionadas (o no descartadas) describiendo sus características principales así como su posible ámbito de utilización en función de las características del entorno urbano (calles y espacios abiertos).

En general, las especies seleccionadas presentan dimensiones geométricas grandes, por lo que la mayoría de ellas son susceptibles de ser utilizadas en calles de anchura como mínimo mediana.

Un análisis cuantitativo de los resultados ofrece los siguientes números de especies utilizables en cada tipología de espacio urbano (Tabla 5). Se indican el número de especies según su tipo de foliación además del número de especies con tolerancia media o alta al calor, el pavimento o la salinidad.

Se obtiene un reducido número de especies utilizables en calles estrechas (por lo que se ha optado por incluir dos especies de hoja perenne) aunque a este respecto conviene indicar que este tipo de calles tiende a presentar valores de AR mayores, por lo que la superficie de calle afectada por el sombreado de las edificaciones es mayor.

En cuanto a las calles de anchura mediana, la cual es una tipología muy frecuente en los núcleos urbanos de la parte oriental de la cornisa cantábrica, se han obtenido para las mismas 13 tipos de especies a las cuales se podrían sumar las 3 especies obtenidas para las calles estrechas al considerarse también adaptables.

Por lo tanto, se obtienen 15 especies de arbolado utilizables en calles de hasta $20 \mathrm{~m}$. de anchura, de las cuales el $80 \%$ presenta una tolerancia al pavimento media o alta, lo que se considera relevante debido a la dificultad de disponer zonas verdes en este tipo de calles.

Se obtienen 20 especies para su utilización en calles anchas o espacios abiertos, de las cuales el 50\% presenta una tolerancia al pavimento media o alta, aunque en este tipo de espacios existe una mayor posibilidad de disponer zonas verdes. En ambos casos resultaría más efectiva la utilización de árboles de grandes dimensiones.

La mayoría de especies seleccionadas son de hoja caduca, aunque también se han incluido algunas especies de hoja perenne para su utilización en calles estrechas y espacios abiertos, ya que éstos últimos suelen presentar superficies lo suficientemente extensas como para que la existencia de áreas sombreadas en los mismos no generen inconvenientes en época invernal, al existir también zonas soleadas.

Respecto a la tolerancia al ambiente salino, el abanico de especies utilizables se reduce casi a la mitad para todas las tipologías de calles, por lo que la elección de especies para áreas cercanas a la costa quedaría aún más restringida.

\subsection{Otros condicionantes a considerar}

A la hora de elegir una especie de arbolado concreta conviene tener en consideración algunas otras características que pueden presentar los espacios urbanos:

- Subregión climática: la vertiente atlántica, en zonas cercanas a la costa, requerirá una mayor adaptación a la salinidad, mientras que para la zona interior se necesitarán mayores tolerancias al calor.

- Distribución funcional de la calle: condicionará el adecuado posicionamiento del arbolado en la sección de la calle. Resulta más apropiada la plantación de arbolado en zonas peatonales, aunque se deberá contemplar la distancia mínima de los elementos de arbolado respecto a las fachadas.

- Orientación de la calle: será determinante para determinar si es procedente disponer el arbolado en uno o a ambos lados de la calle, ya que en una hipotética orientación Este- 
Tabla 4. Especies de arbolado seleccionadas. Características generales.

\begin{tabular}{|c|c|c|c|c|c|c|c|c|c|c|c|}
\hline \multirow{3}{*}{ Nombre botánico } & \multirow{3}{*}{ Nombre común } & \multicolumn{4}{|c|}{ Características Morfológicas } & \multirow{3}{*}{$\begin{array}{c}\text { Características } \\
\text { Fisiológicas }\end{array}$} & \multirow{3}{*}{$\begin{array}{l}\text { Densidad } \\
\text { de } \\
\text { Foliación }\end{array}$} & \multirow{3}{*}{$\begin{array}{c}\text { Ámbito } \\
\text { utilización }\end{array}$} & \multicolumn{3}{|c|}{ Adaptación entorno urbano } \\
\hline & & \multirow{2}{*}{ Forma } & \multicolumn{2}{|c|}{ Altura } & \multirow{2}{*}{$\begin{array}{c}\text { Anchura } \\
\text { Dc.med }\end{array}$} & & & & \multirow{2}{*}{ Calor } & \multirow{2}{*}{ Pavimento } & \multirow{2}{*}{ Salinidad } \\
\hline & & & H.med & H.max & & & & & & & \\
\hline Acer campestre & Arce menor & Esférica & 13 & 20 & 13 & Caduca & Media & Calles medianas & Alta & Alta & Media \\
\hline Acer opalus & Acirón & Cónica & 9 & 15 & 7 & Caduca & Alta & Calles medianas & Alta & Media & Media \\
\hline Acer platanoides & Arce real & Esférica & 15 & 25 & 9 & Caduca & Alta & Calles medianas & Alta & Media & Media \\
\hline Acer pseudoplatanus & Falso plátano & Cónica & 25 & 30 & 15 & Caduca & Media & Espacios abiertos & Baja & Baja & Baja \\
\hline Aesculus hippocastanum & Castaño de Indias & Cilíndrica & 25 & 30 & 20 & Caduca & Alta & Calles anchas & Baja & Baja & Baja \\
\hline Alnus glutinosa & Aliso & Cónica & 18 & 25 & 12 & Caduca & Media & Calles medianas & Baja & Baja & Media \\
\hline Betula alba & Abedul & Esférica & 18 & 20 & 15 & Caduca & Media & Calles medianas & Baja & Baja & Baja \\
\hline Carpinus betulus & Carpe & Cónica & 17 & 30 & 12 & Caduca & Alta & Calles anchas & Baja & Baja & Baja \\
\hline Castanea sativa & Castaño & Cónica & 20 & 30 & 16 & Caduca & Alta & Calles anchas & Alta & Baja & Media \\
\hline Catalpa bignonioides & Catalpa común & Esférica & 10 & 15 & 9 & Caduca & Media & Calles medianas & Alta & Alta & Media \\
\hline Cedrus atlantica & Cedro del Atlas & Cónica & 35 & 40 & 25 & Perenne & Media & Espacios abiertos & Alta & Baja & Baja \\
\hline Celtis australis & Almez & Esférica & 15 & 25 & 14 & Caduca & Alta & Calles medianas & Alta & Alta & Media \\
\hline Cercis siliquastrum & Árbol del amor & Esférica & 5 & 12 & 5 & Caduca & Media & Calles medianas & Alta & Alta & Media \\
\hline Fagus syluatica & Haya & Esférica & 21 & 30 & 21 & Caduca & Alta & Espacios abiertos & Baja & Baja & Baja \\
\hline Fraxinus excelsior & Fresno & Cónica & 30 & 35 & 22 & Caduca & Media & Espacios abiertos & Media & Media & Media \\
\hline Juglans regia & Nogal & Esférica & 20 & 25 & 13 & Caduca & Media & Calles anchas & Media & Media & Baja \\
\hline Ligustrum japonicum & Aligustre & Esférica & 5 & 8 & 5 & Perenne & Alta & Calles estrechas & Media & Media & Media \\
\hline Magnolia grandiflora & Magnolia común & Cónica & 13 & 25 & 9 & Perenne & Alta & Espacios abiertos & Media & Media & Media \\
\hline Phyllirea latifolia & Labiérnago negro & Esférica & 6 & 10 & 6 & Perenne & Media & Calles estrechas & Alta & Alta & Media \\
\hline Platanus hispánica & Plátano común & Esférica & 22 & 35 & 20 & Caduca & Alta & Espacios abiertos & Alta & Alta & Baja \\
\hline Populus alba & Áamo blanco & Cilíndrica & 27 & 30 & 20 & Caduca & Media & Calles anchas & Media & Alta & Baja \\
\hline Prunus domestica & Ciruelo & Esférica & 5 & 10 & 5 & Caduca & Media & Calles estrechas & Alta & Media & Baja \\
\hline Quercus faginea & Roble carrasqueño & Esférica & 12 & 20 & 12 & Caduca & Media & Calles medianas & Alta & Media & Baja \\
\hline Quercusilex & Encina & Esférica & 15 & 35 & 14 & Perenne & Media & Espacios abiertos & Alta & Media & Media \\
\hline Quercus palustris & Roble palustre & Cónica & 20 & 30 & 12 & Caduca & Alta & Calles anchas & Media & Baja & Baja \\
\hline Quercus petraea & Albar & Esférica & 20 & 35 & 20 & Caduca & Alta & Espacios abiertos & Baja & Media & Baja \\
\hline Quercus pyrenaica & Rebollo & Cónica & 15 & 25 & 12 & Caduca & Alta & Calles medianas & Media & Media & Media \\
\hline Quercus robur & Roble & Cilíndrica & 25 & 40 & 14 & Caduca & Media & Espacios abiertos & Media & Alta & Alta \\
\hline Quercus suber & Alcornoque & Irregular* & 15 & 25 & 15 & Perenne & Alta & Espacios abiertos & Alta & Media & Media \\
\hline Sorbus aucuparia & Azarollo & Cilíndrica & 10 & 15 & 5 & Caduca & Alta & Calles medianas & Alta & Baja & Baja \\
\hline Sorbus domestica & Sorbo & Cónica & 15 & 20 & 8 & Caduca & Media & Calles medianas & Alta & Media & Baja \\
\hline Tilia platyphyllos & Tilo común & Cónica & 32 & 40 & 21 & Caduca & Media & Espacios abiertos & Media & Baja & Baja \\
\hline Tilia tomentosa & Tilo plateado & Cónica & 27 & 30 & 17 & Caduca & Alta & Calles anchas & Media & Alta & Media \\
\hline Ulmus glabra & Olmo & Cilíndrica & 35 & 40 & 15 & Caduca & Alta & Espacios abiertos & Baja & Baja & Baja \\
\hline Ulmus minor & Olmo común & Cónica & 25 & 40 & 15 & Caduca & Alta & Espacios abiertos & Alta & Alta & Alta \\
\hline
\end{tabular}

Oeste, el lado Norte de la misma estará sombreada por las edificaciones de manera permanente (29).

- Separación entre elementos: vendrá condicionada por las características morfológicas del arbolado y su adecuación a la distribución funcional de la calle.

\subsection{Recomendación de especies}

Respecto a las características morfológicas, se opta por recomendar especies con forma esférica o cónica, ya que su ca- pacidad de sombreado es superior a las de forma cilíndricaovoidal (19). En cuanto a las características fisiológicas, se considera suficiente que las especies presenten una densidad de foliación media o alta.

También se ha considerado relevante que, dado que el objetivo es la generación de sombra, las especies presenten un grado aceptable de tolerancia al calor, siendo asimismo recomendable una adecuada adaptabilidad de las especies a las zonas pavimentadas.

Tabla 5. Número de especies por ámbito de utilización.

\begin{tabular}{|c|c|c|c|c|c|c|}
\hline & \multirow{2}{*}{ No total } & \multicolumn{2}{|c|}{ Foliación } & \multicolumn{3}{|c|}{ Aptitud al entorno } \\
\hline & & Caduca & Perenne & Calor & Pavimento & Salinidad \\
\hline Calles estrechas & 3 & 1 & 2 & 3 & 3 & 2 \\
\hline Calles medianas & 12 & 12 & $\mathrm{O}$ & 9 & 9 & 7 \\
\hline Calles anchas & 7 & 7 & o & 5 & 3 & 2 \\
\hline Espacios abiertos & 13 & 9 & 4 & 9 & 7 & 6 \\
\hline
\end{tabular}


Tabla 6. Especies recomendadas por ámbito de utilización.

\begin{tabular}{|l|l|c|c|c|}
\hline & & Vertiente atlántica (*) & Zona intermedia & Zona interior \\
\hline Calles estrechas & Ligustrum japonicum & Sí & Sí & SÍ \\
\hline & Prunus domestica & NO & Sí & Sí \\
\hline Calles medianas & Acer platanoides & Sí & Sí & SÍ \\
\hline & Catalpa bignonioides & Sí & SÍ & Sí \\
\hline & Celtis australis & Sí & Sí & Sí \\
\hline & Quercus pyrenaica & Sí & Sí & Sí \\
\hline & Sorbus domestica & Sí & Sí & Sí \\
\hline Calles anchas & Tília tomentosa & NO & Sí & Sí \\
\hline Espacios abiertos & Platanus hispanica & Sí & Sí & Sí \\
\hline & Quercus Ilex & Sí & Sí \\
\hline
\end{tabular}

(*) Consideradas como tales las zonas costeras.
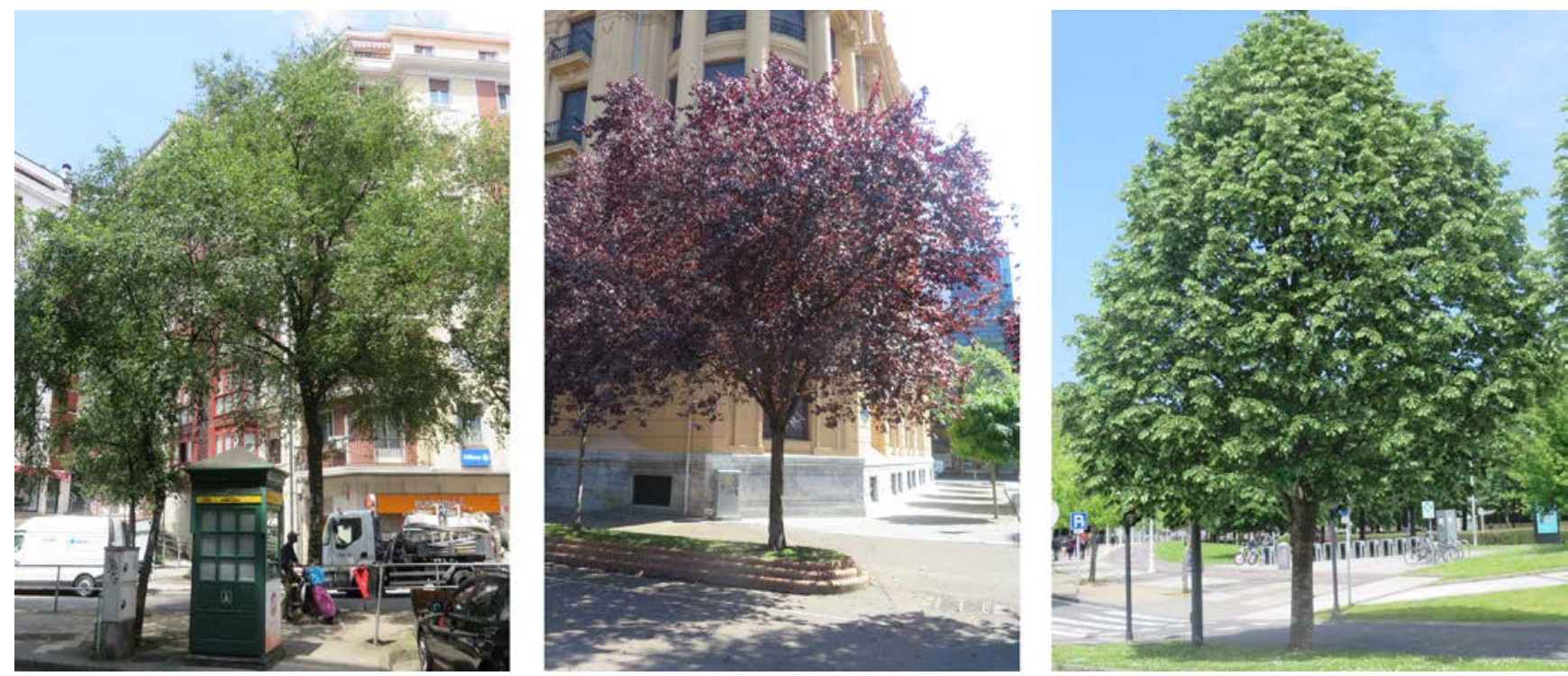

Figura 5. Especies seleccionadas. Betula alba, Prunus domestica y Tilia tomentosa.

En la Tabla 6 se presentan las especies consideradas como más apropiadas para su plantación en los entornos urbanos, determinándose su adaptabilidad a las tres subregiones climáticas de la parte oriental de la cornisa cantábrica:

La mayoría de estas especies se utilizan de manera bastante habitual en los entornos urbanos lo que también se considera indicativo acerca de su idoneidad para las funciones contempladas en el presente estudio. La figura 5 presenta algunos ejemplos de las especies seleccionadas.

\section{CONCLUSIONES}

Según la metodología planteada y el proceso de selección de especies arbóreas, las principales conclusiones que se pueden extraer son las siguientes:

- Se ha obtenido un interesante abanico de especies de arbolado (35 especies en total) utilizables en entornos urbanos para el sombreado. Se han seleccionado especies utilizables en todas las tipologías de calle contempladas.

- Las especies con un mayor volumen de copa proporcionarán una mayor superficie de sombra, por lo que se recomienda la utilización de especies con forma de copa esférica o cónica, aunque su plantación en calles de anchura reducida será más complicada por su posible influencia en las fachadas de los edificios.
- Se observan mayores dificultades para seleccionar especies aptas para calles estrechas, donde sus dimensiones están más limitadas, por lo que se deberá prever un mayor agrupamiento de unidades para la obtención de áreas de sombra más extensivas.

- Las dificultades para integrar el arbolado en calles de anchura reducida lleva a pensar en la posibilidad de una redistribución funcional de la calle según la cual se podría conseguir una más adecuada zonificación respecto a la radiación solar y unas mayores posibilidades de disposición de arbolado.

- 12 de las 35 especies seleccionadas presentan una baja tolerancia a las zonas pavimentadas, donde las tierras presentan una mayor compacidad y existe un menor aporte de agua atmosférica. Por otro lado, 26 de las 35 especies seleccionadas tienen una tolerancia aceptable al calor lo que hace posible su utilización en cualquiera de las tres subregiones climáticas contempladas.

- La mitad de las especies seleccionadas presentan una baja tolerancia a los ambientes salinos por lo que el número de especies utilizables en los entornos urbanos de las zonas costeras es más restringido.

En este estudio se ha propuesto una metodología para la selección de especies de arbolado aptas para el sombreado, lo que no restringe la posible utilización con otros fines de especies no seleccionadas en base a dicha metodología. 
Las propiedades de foliación han sido un factor determinante en la elección de especies, siendo las de hoja caduca las más adecuadas para su utilización en entornos urbanos de zonas climá- ticas donde las necesidades de sombreado se reducen a los períodos estivales. Las características morfológicas han supuesto la principal causa de descarte para las especies no seleccionadas.

\section{REFERENCIAS}

(1) IPPC. Intergovernmental panel on climate change (2014). Mitigation of climate change. Chapter 12: Human settlements, infrastructure, and spatial planning. pp. 923-1000. https://www.ipcc.ch/site/assets/uploads/2018/02/ipcc_ wg3_ar5_chapter12.pdf

(2) Brysse, K., Oreskes, N., O’Reilly, J., Oppenheimer, M. (2013). Climate change prediction: Erring on the side of least drama?. Global Environmental Change, 23(1): 327-337. https://doi.org/10.1016/j.gloenvcha.2012.10.008

(3) Bowler, D.E., Buyung-Ali, L., Knight, T.M., Pullin, A.S. (2010). Urban greening to cool towns and cities: A systematic review of the empirical evidence. Landscape and Urban Planning, 97(3): 147-155. https://doi.org/10.1016/j.landurbplan.2010.05.006

(4) Dimoudi, A., Nikolopoulou, M. (2003). Vegetation in the urban environment: Microclimatic analysis and benefits. Energy and Buildings, 35(1): 69-76. https://doi.org/10.1016/So378-7788(02)00o81-6

(5) Klemm, W., Heusinkveld, B.G., Lenzholzer, S., van Hove, B. (2015). Street greenery and its physical and psychological impact on thermal comfort. Landscape and Urban Planning, 138: 87-98. https://doi.org/10.1016/j.landurbplan.2015.02.009

(6) Nowak, D.J., Crane, D.E., Stevens, J.C. (2006). Air pollution removal by urban trees and shrubs in the United States. Urban Forestry \& Urban Greening, 4(3-4): 115-123. https://doi.org/10.1016/j.ufug.2006.01.007

(7) Wolch, J.R., Byrne, J., Newell, J.P. (2014). Urban green space, public health, and environmental justice: The challenge of making cities 'just green enough'. Landscape and Urban Planning, 125: 234-244. https://doi.org/10.1016/j.landurbplan.2014.01.017

(8) Park, M., Hagishima, A., Tanimoto, J., Narita, K. (2012). Effect of urban vegetation on outdoor thermal environment: Field measurement at a scale model site. Building and Environment, 56: 38-46. https://doi.org/10.1016/j.buildenv.2012.02.015

(9) Grahn, P., Stigsdotter, U.K. (2010). The relation between perceived sensory dimensions of urban green space and stress restoration. Landscape and Urban Planning, 94(3): 264-275. https://doi.org/10.1016/j.landurbplan.2009.10.012

(10) Hartig, T. (2008). Green space, psychological restoration, and health inequality. The Lancet, 372(9650): 1614-1615. https://doi.org/10.1016/So140-6736(08)61669-4

(11) Mayer, H., Hoppe, P. (1987). Thermal comfort of man in different urban environments. Theoretical and Applied Climatology, 38(1): 43-49. https://doi.org/10.1007/BFoo866252

(12) AENOR (2006). Norma UNE-EN ISO 7730:2006- ergonomía del ambiente térmico. Determinación analítica e interpretación del bienestar térmico mediante el cálculo de los índices PMV y PPD y los criterios de bienestar térmico local. Asociación Española de Normalización.

(13) Santamouris, M., Gaitani, N., Spanou, A., et al. (2012). Using cool paving materials to improve microclimate of urban areas - design realization and results of the flisvos project. Building and Environment, 53: 128-136. https://doi. org/10.1016/j.buildenv.2012.01.022

(14) Acero, J.A., Herranz-Pascual, K. (2015). A comparison of thermal comfort conditions in four urban spaces by means of measurements and modelling techniques. Building and Environment, 93: 245-257. https://doi.org/10.1016/j.buildenv.2015.06.028

(15) Lau, K.K., Ren, C., Ho, J., Ng, E. (2016). Numerical modelling of mean radiant temperature in high-density sub-tropical urban environment. Energy and Buildings, 114: 80-86. https://doi.org/10.1016/j.enbuild.2015.06.035

(16) Kong, L., Lau, K.K., Yuan, C., et al. (2017). Regulation of outdoor thermal comfort by trees in Hong Kong. Sustainable Cities and Society, 31: 12-25. https://doi.org/10.1016/j.scs.2017.01.018

(17) Berry, R., Livesley, S.J., Aye, L. (2013). Tree canopy shade impacts on solar irradiance received by building walls and their surface temperature. Building and Environment, 69: 91-100. https://doi.org/10.1016/j.buildenv.2013.07.009

(18) Morakinyo, T.E., Kong, L., Lau, K.K., Yuan, C., Ng, E. (2017). A study on the impact of shadow-cast and tree species on in-canyon and neighborhood's thermal comfort. Building and Environment, 115: 1-17. https://doi.org/10.1016/j.buildenv.2017.01.005

(19) Kotzen, B. (2003). An investigation of shade under six different tree species of the Negev desert towards their potential use for enhancing microclimatic conditions in landscape architectural development. Journal of Arid Environments, 55(2): 231-274. https://doi.org/10.1016/So140-1963(03)0oo30-2

(20) Pinty, B., Gobron, N., Widlowski, J., Verstraete, M. (2003). Allometric Relationships of Selected European Tree Species. Betula pubescens, Fagus sylvatica, Larix decidua, Picea abies, Pinus sylvestris. Ispra (Italy): European CommissionJoint Research Centre.

(21) Hongbing, W., Jun, Q., Yonghong, H., Li, D. (2010). Optimal tree design for daylighting in residential buildings. Building and Environment, 45(12): 2594-2606. https://doi.org/10.1016/j.buildenv.2010.05.019

(22) Brown, R.D., Vanos, J., Kenny, N., Lenzholzer, S. (2015). Designing urban parks that ameliorate the effects of climate change. Landscape and Urban Planning, 138: 118-31. https://doi.org/10.1016/j.landurbplan.2015.02.006

(23) Watson, D.J. (1947). Comparative physiological studies on the growth of field crops: I. variation in net assimilation rate and leaf area between species and varieties, and within and between years. Annals of Botany, 11(41): 41-76. http://www. jstor.org/stable/42907002 
(24) Weiss, M., Baret, F., Smith, G.J., Jonckheere, I., Coppin, P. (2004). Review of methods for in situ leaf area index (LAI) determination: Part II. Estimation of LAI, errors and sampling. Agricultural and Forest Meteorology, 121(1): 37-53. https://doi.org/10.1016/j.agrformet.2003.08.001

(25) Vogt, J., Gillner, S., Hofmann, M., et al. (2017). Citree: A database supporting tree selection for urban areas in temperate climate. Landscape and Urban Planning, 157: 14-25. https://doi.org/10.1016/j.landurbplan.2016.06.005

(26) Morakinyo, T.E., Lam, Y.F. (2016). Simulation study on the impact of tree-configuration, planting pattern and wind condition on street-canyon's micro-climate and thermal comfort. Building and Environment, 103: 262-275. https://doi. org/10.1016/j.buildenv.2016.04.025

(27) Ali-Toudert, F., Mayer, H. (2006). Numerical study on the effects of aspect ratio and orientation of an urban street canyon on outdoor thermal comfort in hot and dry climate. Building and Environment, 41(2): 94-108. https://doi. org/10.1016/j.buildenv.2005.01.013

(28) Qaid, A., Ossen, D.R. (2015). Effect of asymmetrical street aspect ratios on microclimates in hot, humid regions. Int. $J$. Biometeorol., 59(6): 657-677. https://doi.org/10.1007/s00484-014-0878-5.

(29) Theeuwes, N.E., Steeneveld, G.J., Ronda, R.J., Heusinkveld, B.G., van Hove, L.W.A., Holtslag, A.A.M. (2014). Seasonal dependence of the urban heat island on the street canyon aspect ratio. $Q J R$ Meteorol Soc., 140(684): 2197-2210. https://doi.org/10.1002/qj.2289

(30) Spangenberg, J. (2010). Nature in megacities. São Paulo/Brazil - a case study, PhD thesis. Weimar: Bauhaus-Universität.

(31) Aizpuru, I., Catalán, P., Garín, F. (1996). Euskal Herriko zuhaitz eta zuhaisken liburua. Vitoria-Gasteiz: Servicio Central de publicaciones del Gobierno Vasco.

(32) Pérez, F.P. (2003). Euskal Herriko zuhaitzak. Argitaletxea: ADEVE, Asociación para la Defensa de las Especies en Vías de Extinción.

(33) Sabaté, S., Gracia, C.A., Sánchez, A. (2002). Likely effects of climate change on growth of Quercus ilex, Pinus halepensis, Pinus pinaster, Pinus sylvestris and Fagus sylvatica forests in the Mediterranean region. Forest Ecology and Management, 162(1): 23-37. https://doi.org/10.1016/So378-1127(02)0o048-8 